\title{
Homeodomain factor Nkx2-5 controls left/right asymmetric expression of bHLH gene eHand during murine heart development
}

\author{
Christine Biben and Richard P. Harvey ${ }^{1}$ \\ The Walter and Eliza Hall Institute of Medical Research, Royal Melbourne Hospital, Victoria 3050 Australia
}

\begin{abstract}
One of the first morphological manifestations of left/right $(\mathrm{L} / \mathrm{R})$ asymmetry in mammalian embryos is a pronounced rightward looping of the linear heart tube. The direction of looping is thought to be controlled by signals from an embryonic $L / R$ axial system. We report here that morphological $L / R$ asymmetry in the murine heart first became apparent at the linear tube stage as a leftward displacement of its caudal aspect. Beginning at the same stage, the basic helix-loop-helix (bHLH) factor gene eHand was expressed in a strikingly left-dominant pattern in myocardium, reflecting an intrinsic molecular asymmetry. In hearts of embryos lacking the homeobox gene $N k x 2-5$, which do not loop, left-sided $e H a n d$ expression was abolished. However, expression was unaffected in $\mathrm{Scl}^{-1-}$ hearts that loop poorly because of hematopoietic insufficiency, and was right-sided in hearts of inv/inv embryos that display situs inversus. The data predict that $e H a n d$ expression is enhanced in descendants of the left heart progenitor pool as one response to inductive signaling from the $\mathrm{L} / \mathrm{R}$ axial system, and that $e \mathrm{Hand}$ controls intrinsic morphogenetic pathways essential for looping. One aspect of the intrinsic response to $L / R$ information falls under Nkx2-5 homeobox control.
\end{abstract}

[Key Words: $\mathrm{bHLH}_{;}$eHand; dHand; Nkx2-5; NK genes; tinman; homeobox; heart development; heart tube; looping morphogenesis]

Received March 7, 1997; revised version accepted April 11, 1997.

The vertebrate heart is derived from paired mesodermal progenitor populations that ingress through the primitive streak at gastrulation and migrate to the anteriorlateral aspect of the embryo. There they begin cytodifferentiation and fuse at the midline to form a beating tube (Rawles 1943; Rosenquist 1966; DeRuiter et al. 1992). Classical experiments show that cardiac potentiality is acquired within mesoderm during gastrulation (Jacobson and Sater 1988; Gonzalez-Sanchez and Bader 1990; Montgomery et al. 1994) in response to inductive signals from underlying endoderm (Jacobson and Sater 1988; Sugi and Lough 1994; Nascone and Mercola 1995; Schultheiss et al. 1995). Several transcription factor genes are now known to be expressed in early cardiac progenitor cells /Chambers et al. 1992; Komuro and Izumo 1993; Lints et al. 1993; Edmondson et al. 1994; Heikinheimo et al. 1994; Laverrière et al. 1994; Evans et al. 1995; Molkentin et al. 1996), although their individual and cooperative roles in induction, commitment, proliferation, and differentiation remain unclear.

The NK-2 class homeobox gene $N k x 2-5 / C s x$ occupies

${ }^{1}$ Corresponding author.

E-MAIL harvey@wehi.edu.au; FAX 61-3-9347-0852. a key position in the cardiac regulatory hierarchy (Harvey 1996). Nkx2-5 was initially isolated by virtue of its structural homology with the Drosophila homeobox gene tinman (Komuro and Izumo 1993; Lints et al. 1993), essential for specification of heart and visceral muscle progenitors in nascent mesoderm of the fly (Bodmer 1993|. Both tinman and $N k \times 2-5$ are expressed in the heart lineage throughout embryogenesis and are induced by dpp/BMP signaling factors (Frasch 1995; Harvey 1996; Schultheiss et al. 1997), suggesting that the two genes have analogous functions during heart development and that genetic pathways underlying heart formation in distantly related organisms have been conserved (Bodmer 1995; Harvey 1996).

Current evidence implicates $N k \times 2-5$ in control of cardiac commitment, differentiation, and morphogenesis (for review, see Harvey 1996). Ectopic expression of $N k \times 2-5$ in frog embryos leads to an enlarged myocardium, possibly through recruitment into the heart of additional cells from the heart morphogenetic field (Fu and Izumo 1995; Chen and Fishman 1996; Cleaver et al. 1996). Mouse embryos homozygous for a targeted interruption of the Nkx2-5 homeodomain form a beating linear heart tube in which most myogenic genes are ex- 
pressed robustly, although those encoding myosin light chain 2V (MLC2V) (Lyons et al. 1995) and CARP, a nuclear ankyrin-repeat protein (Zou et al. 1997), are severely down-regulated.

Nkx2-5 $5^{-1-}$ hearts also exhibit morphogenetic abnormalities consistent with a block to cardiac looping. Looping is the first morphogenetic event that breaks bilateral symmetry in vertebrate embryos, and one of several left/right (L/R) asymmetries that appear during ontogeny (Yost 1995). It is a critical event in the normal sequence of heart development, establishing tissue relationships necessary for downstream chamber, valve, and vessel formation. In humans, a spectrum of life-threatening cardiac abnormalities results from even subtle perturbations to looping (Burn 1991; Bowers et al. 1996).

The direction of heart looping, normally to the right, is conserved in vertebrates and appears to be determined through an inductive interaction with a $L / R$ axial system (Boterenbrood and Nieuwkoop 1973; Levin et al. 1995; Yost 1995; Hyatt et al. 1996). Several genes are now known to be expressed asymmetrically in the $\mathrm{L} / \mathrm{R}$ axis in various systems, both within the trunk region of the embryo activin receptor IIa, HNF3 $\beta$, cSnR, Shh, nodal/cNR-1, and lefty) and in the heart or its progenitors (cSnR, Flectin, hLAMP1, and JB3-epitope) (Levin et al. 1995; Collignon et al. 1996; Lowe et al. 1996; Meno et al. 1996; Tsuda et al. 1996; Isaac et al. 1997; Smith et al. 1997). Perturbation of the expression of trunk genes results in randomization of heart looping (Levin et al. 1995; Isaac et al. 1997), suggesting that an extrinsic embryonic $\mathrm{L} / \mathrm{R}$ patterning system provides looping information to the heart. In the mouse, the TGF- $\beta$-related cytokines nodal and lefty are expressed in left-sided lateral mesoderm from the time of heart tube formation, and expression is reversed or randomized in embryos carrying mutations affecting laterality (Collignon et al. 1996; Lowe et al. 1996; Meno et al. 1996). nodal and lefty are not expressed extensively throughout the heart itself (Collignon et al. 1996; Meno et al. 1996) making it likely that these factors act inductively and locally in the heart to guide aspects of the looping process.

Intrinsic control of heart looping is poorly understood, as are the cellular processes that drive it. Mechanisms proposed to account for heart looping include $L / R$ differences in proliferation, cellular signaling, contractility and alterations in cell shape, as well as mechanical deformation by a variety of means (Stalsberg 1969, 1970; Manasek et al. 1972; Manasek 1983; Itasaki et al. 1991; Yost 1995). Any model that seeks to explain the intrinsic events of cardiac looping must also account for the ability of the heart to loop apparently normally, but with random situs, when left/right axial signaling is disturbed (Collignon et al. 1996; Hyatt et al. 1996; Lowe et al. 1996; Meno et al. 1996) or inhibited (Levin et al. 1995; Hyatt et al. 1996). In cases of randomized situs, the heart appears to be "agnostic" with regard to laterality and a stochastic event may initiate looping morphogenesis (Burn 1991).

Recently, the transcription factor genes dHand (Hed) Th2) and $e H a n d(H x t / T h 1)$, members of the basic helixloop-helix (bHLH) family (Cross et al. 1995; Cserjesi et al. 1995; Hollenberg et al. 1995; Srivastava et al. 1995!, have been implicated in intrinsic control of cardiac looping. dHand and $e H a n d$ have overlapping expression patterns during murine development: $d H$ and is expressed uniquely in maternal decidua and eHand in trophoblast and extra-embryonic mesoderm, whereas both genes are expressed in heart, lateral mesoderm, and neural crestderived sympathetic trunk and adrenal medulla (Cross et al. 1995; Cserjesi et al. 1995; Hollenberg et al. 1995; Srivastava et al. 1995). An early role for $e H a n d$ in promoting trophoblast differentiation was suggested by enforced expression in mouse blastomeres and a trophoblast cell line (Cross et al. 1995). Important to this study, inhibition of Hand gene expression in cultured chick embryos using antisense oligonucleotides led to arrest of heart development at early looping stages (Srivastava et al. 19951.

In this paper, we report our findings on the gross morphological changes that accompany the earliest stages of cardiac looping in the mouse, and a detailed analysis of the expression of Hand genes in embryonic hearts of normal mice and three genetic models of looping dysmorphogenesis. Hand expression was complex and dynamic, defining novel regionalities in the linear and looping heart tube and surrounding mesoderm. The most significant finding was that $e H$ and came to be expressed in a strikingly left-sided pattern in the looping heart, an observation that betrays an important interface between regulation of Hand gene expression and signaling from the embryonic $L / R$ asymmetry system. We extended these observations to show that left-sided expression of eHand in myocardium was under control of the homeobox gene $N k x 2-5$. The data establish an important regulatory link between a homeobox gene and a bHLH factor in cardiac development, and place both in an intrinsic looping pathway responsive to $L / R$ signaling.

\section{Results}

\section{Early stages of looping morphogenesis}

Morphogenesis of the mammalian heart has been studied over the last decades using histological and electron microscopic techniques (DeHaan 1965; Challice and Viragh 1973; Layton et al. 1980; Kaufman and Navaratnam 1981; Icardo 1984). However, little emphasis has been placed on the caudal region of the heart, in particular, formation of the primitive atrial chamber.

Analysis of gross morphology and histology of heart formation in CBA mice has revealed asymmetric development in the caudal region of the linear heart tube before overt ventricular looping (represented schematically in Fig. 1 and in photomicrographs in Fig. 3, below). The caudal region developed such that there was a clear displacement of the central axis of the heart to the left. In histological sections, this asymmetry could be seen as a leftward positioning of the dorsal mesocardium relative to the midline of the pharynx (Fig. 4D,E, below).

Observation of early heart tube stages has also highlighted the sequential appearance of the inter-ventricular 


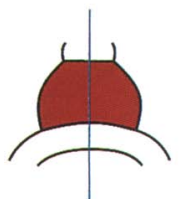

LS-0

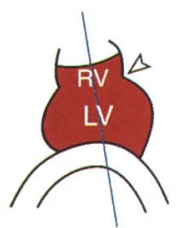

LS-I

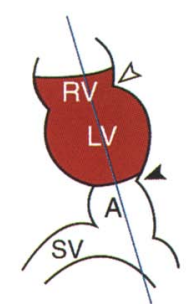

LS-II

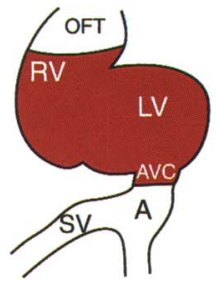

LS-III
Figure 1. Early stages of cardiac looping morphogenesis in CBA mouse embryos. Figure illustrates four stages of morphogenetic progression (LS-0 to LS-III) through early looping stages of heart development. Stages were assigned after inspection of gross anatomy of hearts in paraformaldehyde-fixed whole embryos (see Fig. 3). (LS-0) Near linear (unlooped) heart tube. At this stage, cardiac progenitors are still largely unfused in the dorsal aspect. The A/P axis of the heart is parallel with that of the neural tube and pharynx. (LS-I) Heart tube in which the caudal region displays asymmetric development. The apparent leftward displacement reflects a leftward positioning of the dorsal mesocardium relative to the midline of the neural tube and pharynx (see Fig. 4D,E) and potentially, asymmetrical development of the sinus venosa and surrounding tissues. Because of these asymmetries, the heart tube adopts a tilted $\mathrm{A} / \mathrm{P}$ axis (blue line/ in the left/right plane. The interventricular sulcus is first apparent (open arrowhead), distinguishing future left and right ventricular chambers. There is no overt looping of the ventricular region and a separate atrial chamber is not yet obvious. (LS. II) Heart tube in which a separate atrial chamber and atrioventricular sulcus (solid arrowhead) are first apparent. Looping of the ventricular region is absent or minimal, although the outflow tract may sometimes bend a little toward the left. (LS-III) Heart in which overt ventricular looping is beginning. The atrioventricular canal is beginning to elongate, coincident with appearance of endocardial cushions. Atrial development is asymmetrical, suggestive of the appearance of separate left and right atrial chambers. The left ventricle at this stage is beginning a pronounced expansion, occupying space in front of (ventral to) the atrial chamber and left sinus venosus. (A) Atrium; $(A V C)$ atrioventricular canal; (LV) left ventricle; (OFT) outflow tract; $(\mathrm{RV})$ right ventricle; (SV) sinus venosa.

and atrio-ventricular (A/V) sulci, as well as the atrial chamber and $\mathrm{A} / \mathrm{V}$ canal. Because heart development is not strictly synchronous with other developmental processes such as somite formation (Kaufman and Navaratnam 1981), we have classified the onset of heart looping into four progressive stages [looping stages (LS) 0-III], according to appearance of landmarks described above (Fig. 1).

\section{Hand gene expression}

Using in situ hybridization of digoxygenin-labeled cRNA probes and a whole-mount protocol, expression of $e$ Hand and $d$ Hand were examined in embryonic day 7.5 (E7.5)E11.5 embryos with emphasis on cardiac progenitors and hearts. These studies complement and extend patterns described previously (Cross et al. 1995; Cserjesi et al. 1995; Hollenberg et al. 1995; Srivastava et al. 19951, in which regional expression of $e H a$ and in the mouse heart was noted (Cserjesi et al. 1995; Hollenberg et al. 1995), but not characterized in detail. Expression of Hand genes was both complex and dynamic, and, whereas individual patterns overlapped, they also showed striking differences (see below).

\section{eHand expression-general pattern}

At early headfold stages, $e H$ and was transcribed strongly in extra-embryonic mesoderm of the yolk sac, as well as in ectoderm and mesoderm of the amnion. Initially, expression in the embryo proper was restricted to cells at the junction between embryonic and extra-embryonic tissue (see below). By E8.5, however, eHand was expressed in myocardial and pericardial layers of the heart, as well as in lateral mesoderm, more caudally (Fig. 2BD). Removal of the pericardium from E9.5 embryos revealed two $\mathrm{eHand}$ expression domains in myocardiumone cranial, the other caudal, separated by a nonexpressing region (Fig. 2C). Expression in the caudal domain was

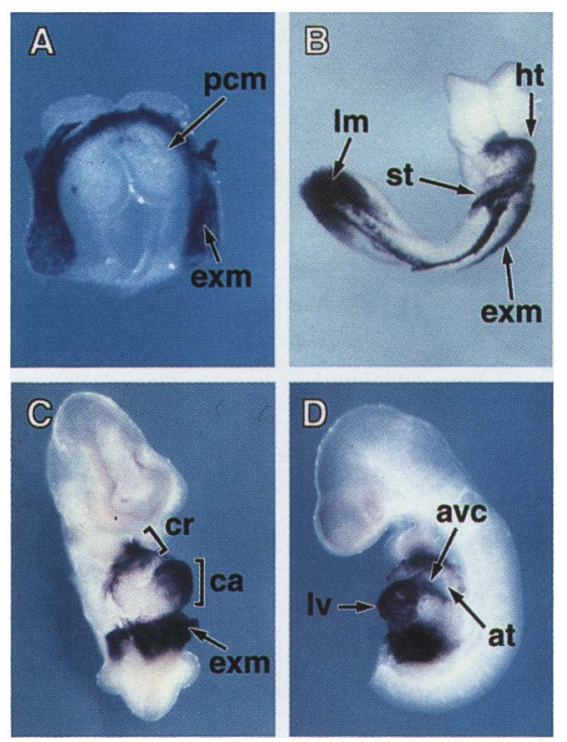

Figure 2. eHand expression in embryos analyzed by wholemount in situ hybridization. $(A)$ Early foregut pocket stage embryo (ventral view) showing expression in extra-embryonic membranes. Precardiac mesoderm is largely negative (see Fig. 5 and text $\mid\langle B|$ E8.5 embryo showing asymmetric expression of $e H a n d$ in the heart and pericardium, as well as expression in septum transversum, lateral mesoderm, and remnants of dissected extra-embryonic membranes. $(C, D)$ E9.5 embryo (ventral and left aspects, respectively) showing expression in the heart region after removal of the pericardial membranes and the caudal trunk and tail. Note separate cranial and caudal domains of $e$ Hand expression $|C|$, left-sided expression in the caudal domain $|C|$ and a transient tongue of $e H a n d$ expression that extends from the left ventricle along the left and front side of the atrial chamber (D). (at) Atrium; (avc) atrioventricular canal; (ca) caudal $e H$ and expression domain; (cr) cranial $e H a n d$ expression domain; (exm) extra-embryonic mesoderm; (ht) heart; (lm) lateral mesoderm; (lv) left ventricle; $(\mathrm{pcm})$ precardiac mesoderm. 
Figure 3. eHand expression in linear and looping hearts analyzed by whole-mount in situ hybridization. Expression in myocardium is revealed after removal of pericardial membranes. All embryos depicted show $\mathrm{H} H$ and expression, except in $C$, showing $M L C 2 V$ expression. Features of heart development in these and similar embryos have been used to assign looping stage desig. nations (see Fig. 1). (A) Linear heart tube embryo (LS-0) showing bilateral $e$ Hand expression in the caudal heart and sinus venosa. $\mathrm{R} \leftrightarrow \mathrm{L}$ indicates the left/right axis. (B) Left view of LS-0 embryo showing that $e H a n d$ expression in the caudal heart is strictly ventral. $\mathrm{D} \leftrightarrow \mathrm{V}$ shows the dorsoventral axis. $(C)$ LS-0 embryo showing MLC2V expression in both dorsal and ventral aspects of the heart tube. $(D)$ LS-1 embryo showing bilateral eHand expression in the caudal heart and sinus venosa. Note the leftward displacement of the caudal heart. Arrow indicates the apparently enhanced expression of $e H a n d$ along the left side of the caudal heart tube. $\{E, F\rangle$ LS-II embryo showing ventral and caudoventral views, respectively. The ventricular region shows a slight asymmetry in this embryo. Note the clearly separate atrial chamber and atrioventricular sulcus. (G) LS-III embryo showing overt ventricular looping and $e H a$ and expression in the left side of the left ventricle. Note the elongating atrioventricular canal and diminished expression in atria and sinus venosa. $(H, I)$ Dissected hearts at E10.5 and E11.5, respectively, showing eHand expression on the left side of the left ventricle and, at E11.5 only, expression on the right side of the right ventricle and outflow tract. (at) Atrium; (avc) atrioventricular canal; (avs) atrioventricular sulcus; (ivs) interventricular sulcus; (lv) left ventricle $_{;}$(oft) outflow tract; $(\mathrm{p})$ pericardium; $(\mathrm{rv})$ right ventricle; (sv) sinus venosa.

left-sided, covering only a portion of the prospective left ventricle, A/V canal, and atrium (Fig. 2C,D), a pattern never observed with other regional cardiac markers (Lyons 1994).

\section{eHand expression in the linear and looping heart tube}

The caudal $e H a n d$ expression domain first became obvious during heart tube fusion (Figs. 2A and 3A). In LS-0 embryos, expression in this domain was bilateral and symmetrical (Fig. 3A), in contrast with the later leftsided expression. However, expression at this early time was restricted to the ventral aspect of the myocardium and sinus venosa, demonstrating for the first time a dorsoventral patterning in the heart tube (Figs. $3 \mathrm{~B}$ and $4 \mathrm{H}$ ). This restriction did not reflect a dorsoventral maturational gradient within myocytes, because $M L C 2 V$, an early myogenic marker, was robustly expressed in both dorsal and ventral aspects (Fig. $3 \mathrm{C}$ ).

Bilateral eHand expression continued in hearts that showed the first evidence of morphological asymmetry in the $\mathrm{L} / \mathrm{R}$ axis (LS-I). However, in some embryos, enhanced $e H a n d$ expression was evident along the left wall of the heart tube, hinting that the left-sided pattern discussed above initially arose superimposed on a bilateral pattern (Fig. 3D and see below). Bilateral expression was still apparent in LS-II embryos (Fig. 3E,F), although at the onset of overt looping (LS-III; Fig. 3G), the bilateral mode appeared to fade in sinus venosa and atrium, superseded by a now stronger left-dominant pattern. This trend con-
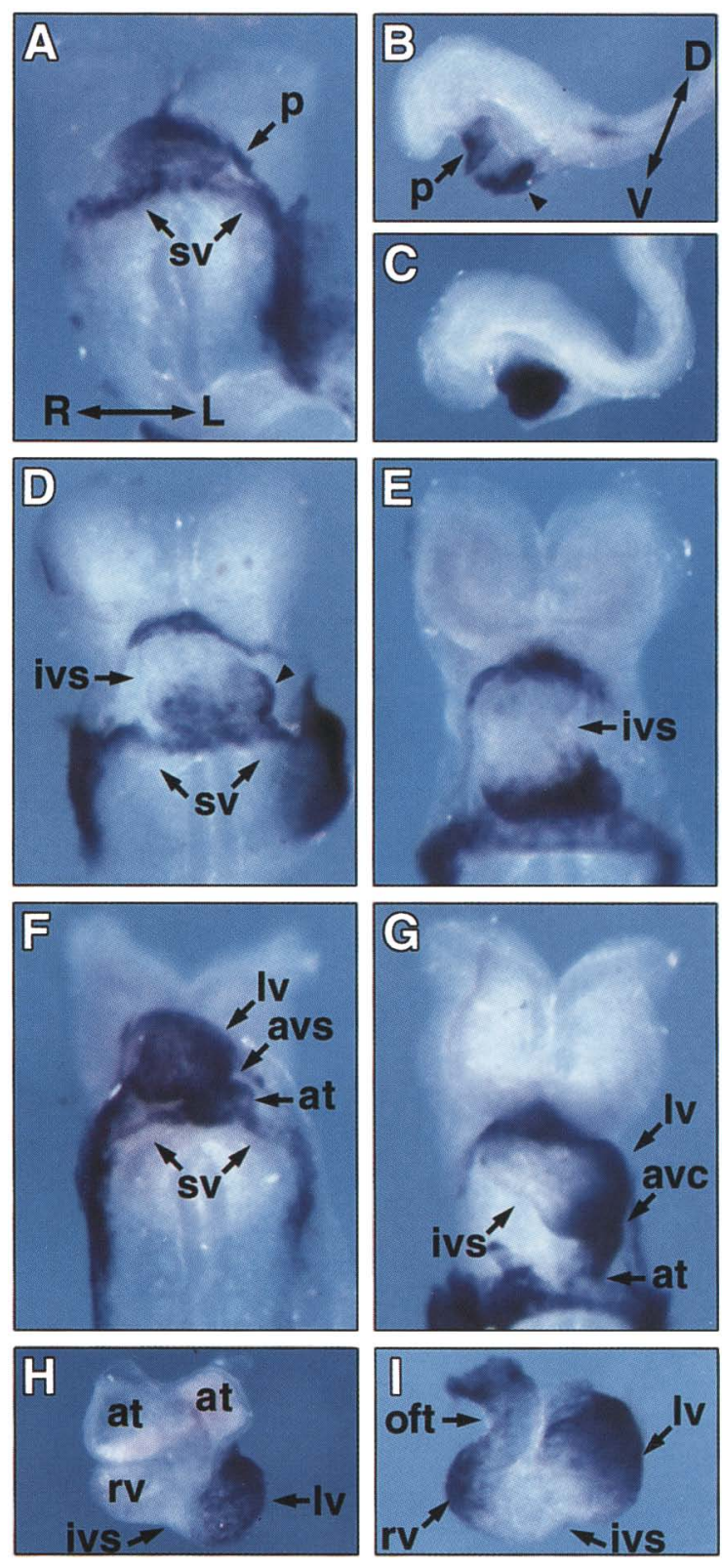

tinued, with bilateral expression lost almost completely by E9.0-E9.5 (Figs. 2D, 4I, and 8A, below).

These experiments did not resolve satisfactorily whether the transition from bilateral to left-sided was passive or active, and therefore whether left-sided $e H$ and expression represents a genuine intrinsic cardiac asymmetry. A left-sided pattern, for example, could be generated passively if the bilateral, ventrally restricted pattern was deployed to the left by the morphogenetic movements of looping (leftward displacement or torsion), although this is not the case in the chick where there is an opposite rightward torsion at an equivalent stage (Stalsberg 1969|.

Serial transverse sectioning of whole-mount embryos shed further light on this issue. In LS-II embryos $(n=3)$, a strikingly asymmetric pattern was evident (Fig. 4A-G). Superimposed on the bilateral pattern (Fig. 4E,F) was a 

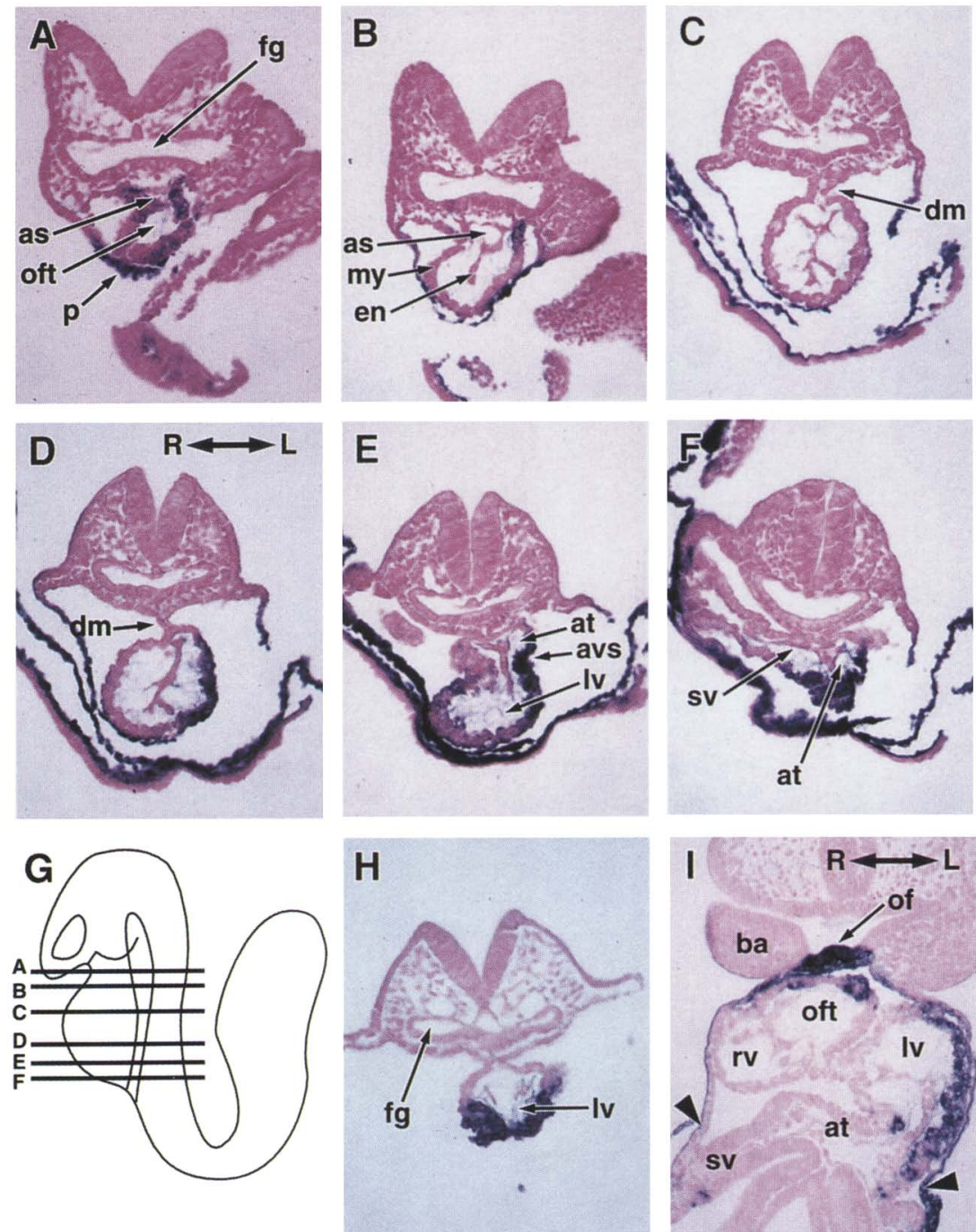

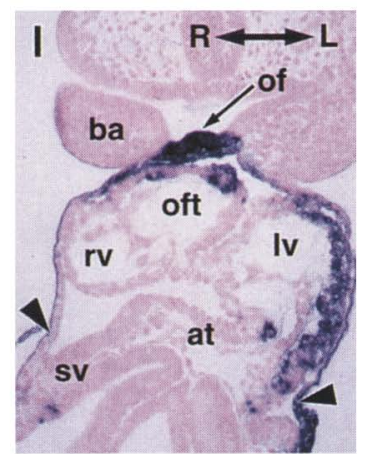

Figure 4. eHand expression in linear and looping hearts, analyzed by histological sectioning of embryos after whole-mount in situ hybridization. $|A-F|$ Selected serial transverse sections of an LS-II embryo showing eHand expression in the heart tube. $\mathrm{R} \leftrightarrow \mathrm{L}$ denotes the left/right axis. Note the distinct cranial $|A, B|$ and caudal $(D-F)$ expression domains, and enhanced expression on the left side of the heart tube in the caudal domain. $\{G \mid$ Diagram of the planes of section shown in $A-F$. $(H)$ Section of an LS-I embryo (pericardium removed) showing that $e$ Hand expression in the caudal domain of the heart tube is strictly ventral. $|I|$ Transverse section through an E8.5 embryo (same embryo as depicted in Fig. 2B), showing eHand expression in oral cavity floor, pericardium, outflow tract myocardium, and the left side of the left ventricle and atrium. $\mathrm{R} \leftrightarrow \mathrm{L}$ denotes the left/right axis. Arrowheads point to pericardial mesoderm on the left and right sides, highlighting enhanced eHand expression on the left. (as) Aortic sac; (at) atrium; |avs) atrioventricular sulcus; (ba) first branchial arch; $(\mathrm{dm})$ dorsal mesocardium; (en) endocardium; (fg) foregut; (lv) left ventricle; (myl myocardium; (of) oral cavity floor; (oft) outflow tract; (p) pericardium; $|\mathrm{rv}|$ right ventricle; $|\mathrm{sv}|$ sinus venosa. zone of considerably enhanced $e$ Hand expression along the left side (Fig. 4D,E). This left-sided pattern extended more dorsally than the bilateral pattern, and in some sections, left-sided expression was clearly separable from bilateral (Fig. 4D). These findings strongly suggest that the left-sided pattern arises from within the bilateral pattern, rather than being generated by rearrangement of the earlier expression through torsion or repression on the right side.

Sectioning of well looped hearts (LS-III; $n=6$ ) revealed $e H a n d$ expression in the left and left-frontal portion of the left ventricle, and on the left side of the atrium (Fig. 4I). Asymmetrical expression in developing atrium after bilateral expression had faded there, was seen clearly in these sections, as well as in whole mounts (Fig. 2D; see Discussion).

We conclude that there are two modes of eHand expression in the caudal domain-bilateral, which ultimately fades in both atria and left ventricle, and leftsided, which begins in the ventricle, common atrium, and sinus venosa of the linear tube before becoming dominant in left ventricle. The latter expression reflects an intrinsic cardiac asymmetry.

\section{Cranial domain of eHand expression}

The cranial eHand expression domain initially comprised oral cavity floor and myocardium (Figs. 4A,B,I). Expression in cranial myocardium was patchy and restricted to the aortic sac region throughout looping stages (Fig. 4A). In later development, as seen in hearts removed from E10.5-E11.5 embryos (Fig. 3I), this expression domain expanded into the bulbus cordis (prospective right ventricle). It is of note that this latter expression occurred predominantly on the right side of the myocardial tube (see Discussion).

We have examined the onset of $e H$ and expression in its cranial domain, comparing it with that of the homeobox gene $N k x 2-5$, a marker of precardiac mesoderm (Lints et al. 1993). Before foregut pocket formation, eHand expression could be detected in ectoderm immediately anterior and anterior-lateral to headfold neurec- 

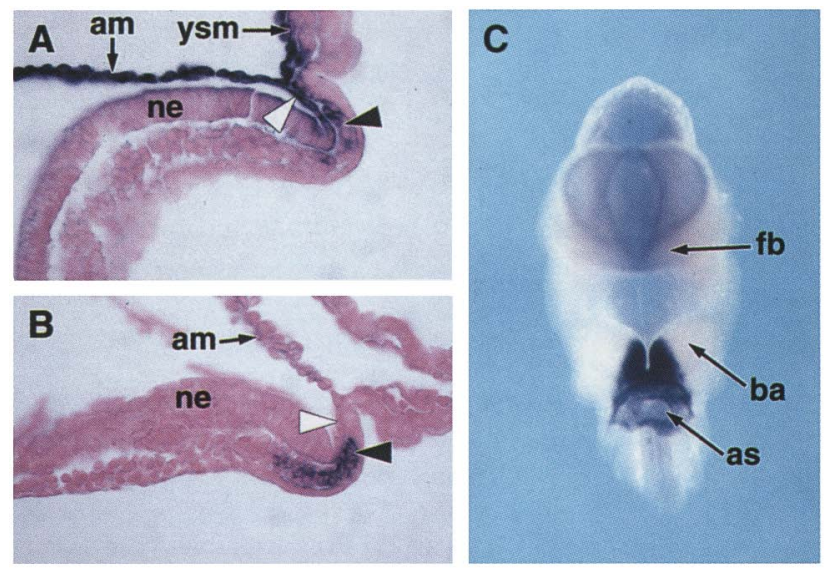

Figure 5. Onset of $e H a n d$ expression in anterior mesectoderm and branchial arches. $|A, B|$ Parasaggital sections of late headfold stage embryos (-E7.5-E8.0) hybridized with eHand and Nkx2-5 probes, respectively. eHand is expressed in ectoderm anterior to the neural plate (white arrowhead) and in underlying mesoderm (solid arrowhead). Nkx2-5 is also expressed in mesoderm underlying the anterior ectoderm, and more broadly within precardiac mesoderm. Note that $e H a n d$-positive ectoderm is contiguous with the ectoderm of the amnion, also strongly positive. $|C|$ Frontal view of the head of an E10.5 embryo dissected from the trunk at the level of the aortic sac. eHand is expressed strongly in the medial portion of the first pair of branchial arches. Signal in brain vesicles is attributable to probe trapping. $|\mathrm{am}|$ Amnion; (as) aortic sac; (ba) first branchial arches; (fb) forebrain; (ne) neurectoderm; (ysm) yolk sac mesoderm.

toderm, the region from which oral cavity is thought to arise (Fig. 5A). Mesoderm underlying this eHand-positive ectoderm also expressed $e H$ and (Fig. $5 \mathrm{~A}$ ), as well as $N k \times 2-5$ (Fig. $5 \mathrm{~B}$ ), although the $N k \times 2-5$ domain extended more laterally and caudally. Therefore, eHand is expressed in only the most anterior precardiac cells at this time. It seems likely that these $e H a n d$-positive ectodermal and precardiac cells give rise to the oral floor and aortic sac cells that express $e H a n d$ in heart tubes of later embryos.

\section{Other sites of eHand expression}

With time, eHand expression in its cranial domain expanded into neighboring tissues. By E9.0-E9.5, expression in oral cavity ectoderm had diminished. However, expression began at this time in mesenchyme of the first pair of branchial arches-initially medially, where in direct contact with the oral floor, then, by E10.5, more laterally as well (Fig. 5C). Eventually, a large proportion of first arch mesenchyme and its epithelium expressed eHand. Around E9.0, eHand was expressed in thyroid diverticulum, a derivative of pharyngeal floor endoderm, at the level of the aortic sac (see below and Fig. 8C). As noted above, by E10.0, expression in aortic sac had expanded along the right side of the bulbus cordis.

A constant feature of the $e$ Hand pattern in linear and looping hearts was expression in pericardium and sep- tum transversum (Fig. 2B). The pericardium has two components, an outer ectodermal layer and an inner mesodermal layer. The ectodermal layer is contiguous with the floor of the oral cavity and expressed $e$ Hand (Fig. 4I). The mesodermal layer, derived from the somatopleure, lines the whole pericardial cavity and is contiguous with dorsal mesocardium and caudally, septum transversum. $e H a n d$ was expressed in ventral but not dorsal pericardial mesoderm (Fig. 4A), mirroring the ventral restriction in myocardial expression.

Pericardial mesoderm may also be modified by signals that govern $\mathrm{L} / \mathrm{R}$ asymmetry. In looping stage embryos, eHand expression in pericardial mesoderm was more pronounced on the left side, seen both in whole mounts (Fig. 2B) and in sections (arrows in Fig. 4I). Therefore, eHand appears to be responsive to laterality signals in both pericardium and myocardium.

\section{dHand expression-onset and general pattern}

$d H a n d$ was first expressed in headfold stage embryos throughout the lateral mesoderm (Fig. 6A). Both splanchnic and somatic layers expressed the gene (Fig. 6B). In serial sections through the precardiac region, the intensity of signal was even at different anteroposterior $(\mathrm{A} / \mathrm{P})$ levels, a situation that changed rapidly on heart tube
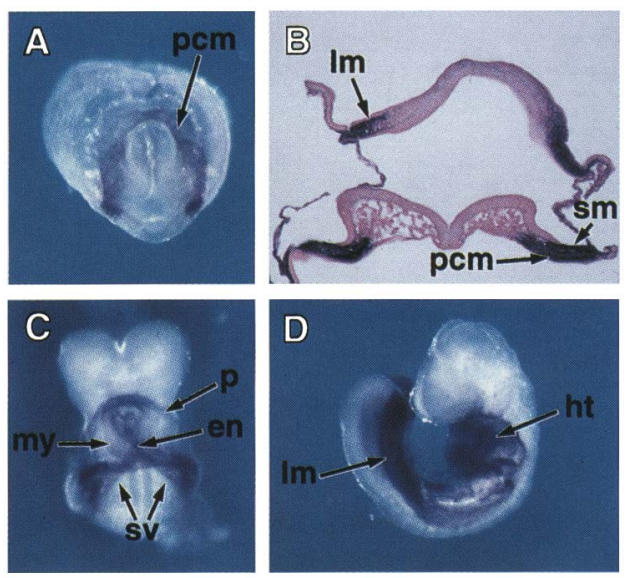

Figure 6. dHand expression in embryos analyzed by wholemount in situ hybridization. (A) Early foregut pocket stage embryo (ventral view) showing $d$ Hand expression in precardiac mesoderm. Lateral plate mesoderm more caudally is also positive, although out of focus in this photomicrograph. $(B)$ Section of the embryo shown in $A$, which passes through precardiac mesoderm (posterior region) and lateral plate mesoderm more caudally. Note $d H$ and expression in both splanchnic (precardiacl and somatic mesoderm. The coelom, which normally separates these layers, has collapsed. $(C, D)$ E8.25 (LS-I; ventral view) and E9.5 (left view) embryos, respectively, showing $d H$ and expression in a complex pattern in the heart region and lateral mesoderm more caudally. In $C$, note strong expression in endocardium and the tilted $A / P$ axis of endocardial tube. (en) Endocardium; $(\mathrm{ht})$ heart; $(\mathrm{lm})$ lateral mesoderm; $(\mathrm{my})$ myocardi$\mathrm{um}_{;}(\mathrm{p} \mid$ pericardium; $(\mathrm{pcm})$ precardiac mesoderm; $(\mathrm{sm})$ somatic mesoderm; (sv) sinus venosa. 
formation (see below). At E8.25 and E9.5, whole-mounts revealed $d H a$ and expression in a complex pattern in the heart region, as well as in lateral mesoderm, more caudally (Fig. 6C,D). As shown below, dHand expression in the heart could be distinguished clearly from that of eHand.

\section{$\mathrm{dHand}$ expression in the linear and looping heart}

Serial transverse sectioning was performed on embryos with linear hearts (LS-0; $n=3$ ) after hybridization with a $d H a n d$ probe (Fig. 7A-G). $d$ Hand was expressed strongly and evenly in endocardium throughout the length of the heart tube. Expression was also seen in all myocardial cells, as well as in dorsal pericardial mesoderm and dorsal mesocardium. A graded distribution of $d H$ and transcripts was evident in myocardium and dorsal pericardial mesoderm, with highest levels at the cranial end.

During looping stages, the gradient of $d H a n d$ transcripts became less apparent as expression faded in caudal regions of the heart tube. By E9.0, it was largely restricted to the outflow tract (Fig. $7 \mathrm{H}$ ), and by E9.5 to the aortic sac (Fig. 7I). Overstaining of embryos revealed low expression remaining across the whole looped heart tube with somewhat higher levels in sinus venosa and septum transversum (Fig. 7I). Expression in outflow tract was predominantly on the right side of the myocardial tube (Fig. $7 \mathrm{H}$ ), similar to the late phase of $e$ Hand expression in the same region (Fig. 3I). Slightly enhanced $d H a n d$ expression was also noted on the left side of the left ventricle (Fig. 7I), resembling the strong left-sided expression of $e$ Hand discussed above.

At later stages, dHand was expressed extensively within mesoderm cranial to the heart, including first arch mesenchyme and that surrounding the aorta, then later in tongue and mandible, as described previously (Cserjesi et al. 1995; Hollenberg et al. 1995).

\section{eHand expression in mutant embryos}

To address the role of Hand genes in looping morphogenesis, we examined their expression in embryos from three mouse strains carrying mutations affecting cardiac looping. In embryos homozygous for a targeted mutation in the homeobox gene $N k \times 2-5$, cardiac looping is blocked and heart morphogenesis does not progress beyond the linear tube stage (Lyons et al. 1995). In these hearts, the early bilateral $e H$ and expression domain appeared to be established normally, although weakly (Fig. 8B,E,F). In contrast, the strong left-sided expression was abolished totally (Fig. 8A-C), as was that in aortic sac and oral cavity floor (Fig. 8C). Expression was unaffected in pericardium and septum transversum (Fig. 8A; data not shown), sites that never express $N k x 2-5$. Expression in thyroid diverticulum was also unaffected, although this structure does express $\mathrm{Nk \times 2}-5$ from an early time (Lints et al. 1993). We conclude that the myocardium of Nkx2-5 mutant embryos is competent to express eHand, but cannot interpret signals that enhance expression on
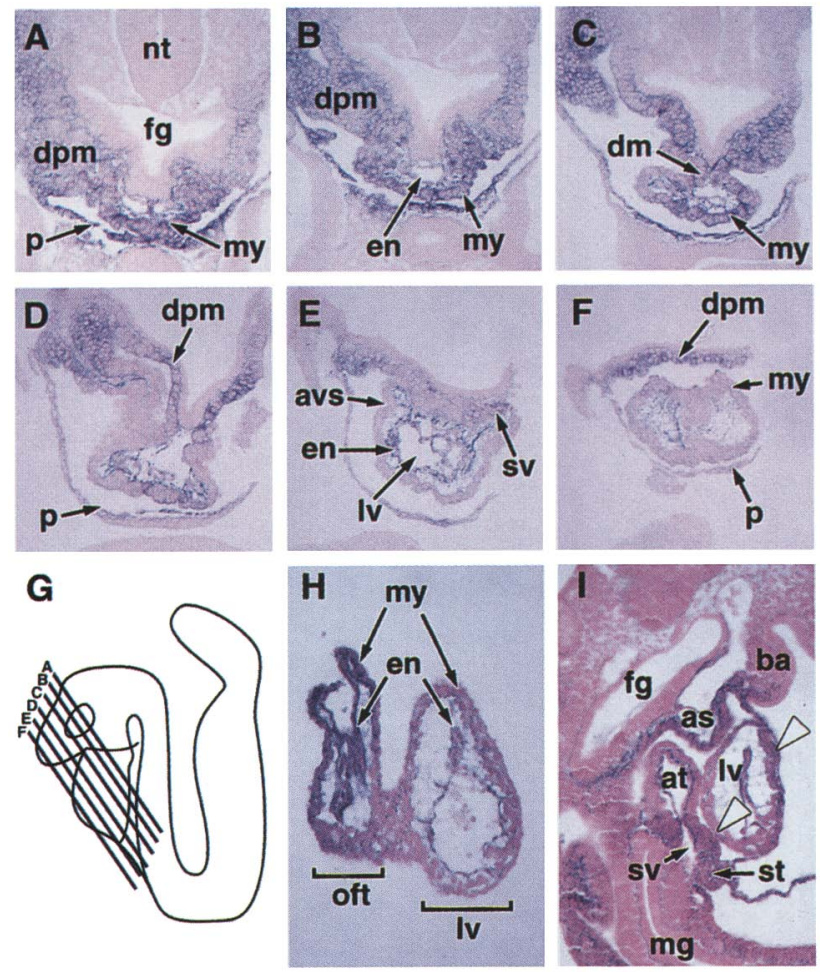

Figure 7. $d$ Hand expression in linear and looping hearts. $(A-F)$ Selected serial transverse sections of an LS-I to LS-II embryo after hybridization in whole-mount with a dHand probe. A graded pattern of $d H$ and expression is apparent in myocardium, pericardium, and dorsal pericardial mesoderm, with highest levels at the cranial end. Endocardium is also positive. $(G)$ Diagram of the planes of section shown in $A-F$. $\{H\}$ Section of a dissected E9.0 heart hybridized in whole mount with a dHand probe, showing expression predominantly in endocardium and outflow tract myocardium. (I) Detail of parasaggital section, centered on the heart region, through an E9.5 embryo hybridized in wholemount with a $d H a n d$ probe. Highest levels of $d H$ and transcripts are seen in the aortic sac, septum transversum, and sinus venosa. Lower levels are seen in atrium and ventricles. Open arrowheads point to the dorsal and ventral walls of the left ventricle. Enhanced $d H$ and expression is seen on the ventral (frontall side. dHand expression can also be seen beginning in the first branchial arch. (at) Atrium; (as) aortic sac; (avs) atrioventricular sulcus; (ba) branchial arch; (dm) dorsal mesocardium; $|\mathrm{dpm}|$ dorsal pericardial mesoderm; (en) endocardium; $(\mathrm{fg})$ foregut; $(\mathrm{lv})$ left ventricle (mg) midgut; (my) myocardium; (nt) neural tube; (oft) outflow tract; $(\mathrm{p})$ pericardium; $\{\mathrm{st}\}$ septum transversum; $|\mathrm{sv}|$ sinus venosa.

the left side of the heart and that are dependent on Nkx2-5 [see Discussion!.

$e H$ and expression was frequently observed ectopically in Nkx2-5 mutants in pharyngeal floor endoderm lying adjacent to the caudal region of the heart (Fig. $8 \mathrm{~B}, \mathrm{E}, \mathrm{F})$. Here, the pharyngeal floor transiently supports the dorsal wall of the atrial chamber and sinus venosa, where there is no myocardial layer (Fig. 8E,F). Because Nkx2-5 is normally expressed in caudal pharyngeal floor during heart tube formation (Lints et al. 1993), the encoded ho- 

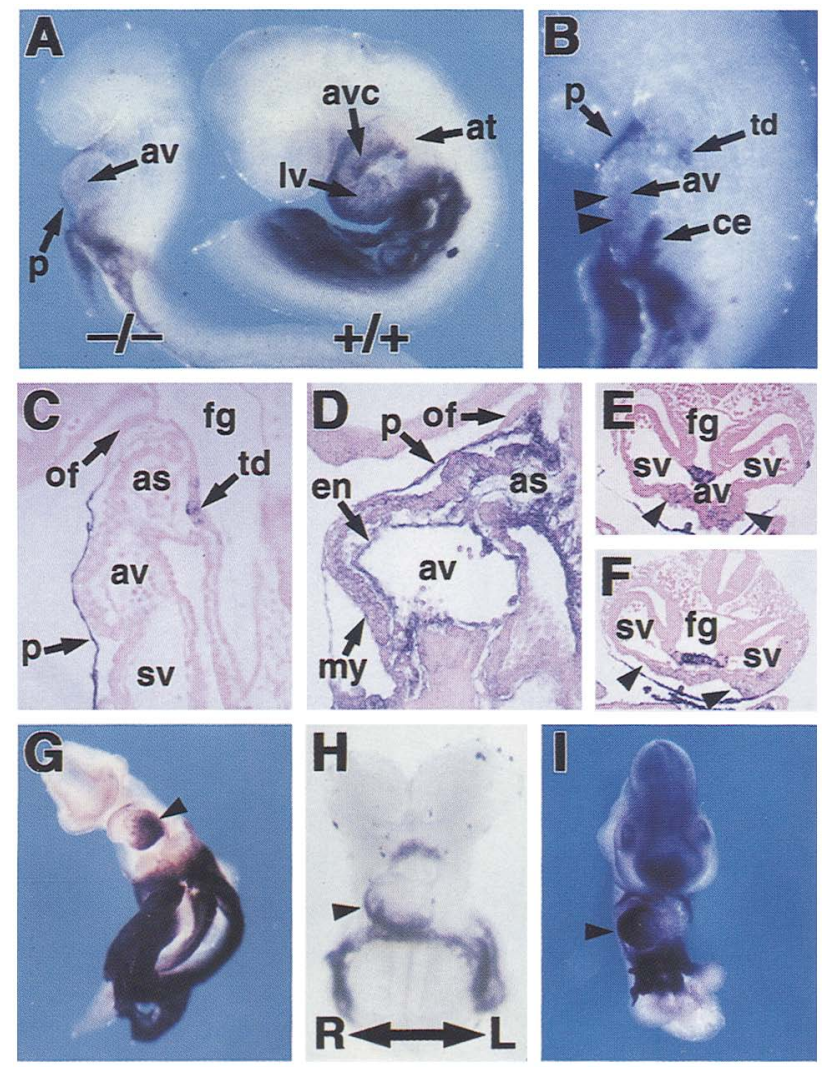

Figure 8. Whole-mount in situ hybridizations showing expression of eHand and dHand in mutant embryos. $|A|$ eHand ex pression in an Nkx2-5 mutant $(-/-)$ and wild-type sibling $|+|+\mid$ at E9.5. (B) Close-up of an Nkx2-5 mutant showing weak midline expression in the myocardium (arrowheads) and normal (thyroid diverticulum) and ectopic (caudal foregut endoderm) expression in pharyngeal floor (see E,F). (C) Section of $N k x 2-5$ mutant hybridized to $e H a n d$ probe. Note expression in pericardium and thyroid diverticulum, but not myocardium. Ectopic eHand expression occurs in the caudal pharynx of this embryo (see $B, E, F)$, but is not evident in this particular section. (D) Section of Nkx2-5 mutant hybridized to $d H$ and probe. Note normal graded expression in myocardium, as well as pericardium (dorsal and ventral) and endocardium. $(E, F)$ Transverse sections of the Nkx2-5 mutant embryo in $B$ showing ectopic $e H a n d$ expression in pharyngeal floor at its cranial (atrioventricular) and more caudal (sinus venosa) levels, respectively. Expression in this region has never been observed in wild-type embryos. Note weak bilateral $e H a n d$ expression in myocardium (arrowheads), but no left-sided expression. $(G) \mathrm{Scl}^{-1-}$ embryo at E9.5 hybridized with eHand, showing strong left-sided expression in the looping heart. $(H, I)$ inv/inv embryos at E8.25 (LSI-LSII) and E9.5, respectively, hybridized to an $e H a n d$ probe. Right-sided expression of eHand is marked by arrowhead. Signal in brain vesicles is attributable to probe trapping. $\mathrm{R} \leftrightarrow \mathrm{L}$ indicates the $\mathrm{L} / \mathrm{R}$ axis. (av) Atrioventricular chamber; (as) aortic sac; (at) atrium; (avc) atrioventricular canal; (ce) caudal foregut endoderm; (en) endocardium; $\{\mathrm{fg}\}$ foregut; $\{\mathrm{lv}\}$ left ventricle; $\{$ my $\}$ myocardium; (of $\}$ oral cavity floor; (p) pericardium; (sv) sinus venosa; (td) thyroid diverticulum.

meodomain factor may actively repress $e H a n d$ expression in this region (see Discussion).
Morphogenesis in the mammalian heart may be affected profoundly by poor cardiac function or nutrition. To test whether loss of left-sided eHand expression in Nkx2-5 mutant hearts was a secondary consequence of local conditions within a dysfunctional heart, expression was examined in embryos homozygous for a targeted mutation in the bHLH gene $\mathrm{Scl}$. Scl is essential for blood formation in the yolk sac, but is not expressed in myocardium. In $\mathrm{Scl}^{-1-}$ embryos, heart looping is abnormal or retarded, presumably a secondary consequence of the haematopoietic deficiency. eHand expression in both its cranial and caudal domains was normal in these embryos (Fig. 8G; data not shown). This result suggests that the loss of eHand expression in Nkx2-5 mutant hearts reflects a genuine regulatory link between the two genes.

Finally, embryos homozygous for the inv transgenic insertion were examined for eHand expression. inv/inv embryos show a highly penetrant reversal of organ situs and right-sided expression of nodal and lefty in lateral mesoderm (Collignon et al. 1996; Meno et al. 1996). At early heart tube stages, we observed a rightward shift in the caudal heart, and evidence of right-sided eHand expression overlapping bilateral expression in the caudal domain (Fig. 8H). At E9.5, eHand was expressed strongly on the right side of the ventricular region (Fig. 8I). These characteristics are the complete opposite of normal, but in accordance with the near-perfect reversal of heart situs seen in this strain (Fig. 8I).

\section{dHand expression in mutant embryos}

In contrast with $e H$ and, $d H$ and was expressed normally in Nkx2-5 mutant hearts. In sections of E8.5 mutants hybridized with the $d H$ and probe, expression was seen in pericardium, endocardium, myocardium, and septum transversum (Fig. 8D). Therefore, $d$ Hand does not fall under Nkx2-5 control. As expected, $d H a n d$ was also expressed normally in $\mathrm{Scl}^{-1-}$ mutant hearts (data not shownl.

\section{Discussion}

We have performed a detailed expression analysis of the bHLH genes eHand and $d H a n d$ in developing murine hearts, and in those carrying mutations affecting cardiac looping. Our findings demonstrate a genetic interaction between the homeobox gene Nkx2-5 and $\mathrm{HHand}$ within an intrinsic cardiac pathway responsive to laterality signals. They also implicate this pathway in genetic control of cardiac looping.

\section{eHand expression}

eHand was expressed in separate cranial and caudal domains in the linear and looping heart tube /summarized in Fig. 91. The cranial domain, initially patchy, appears to derive from very early expression in precursors of the oral cavity and subjacent precardiac mesoderm.

In its caudal domain, $e H$ and was initially expressed in forming ventricle, atrium, and sinus venosa. This early 

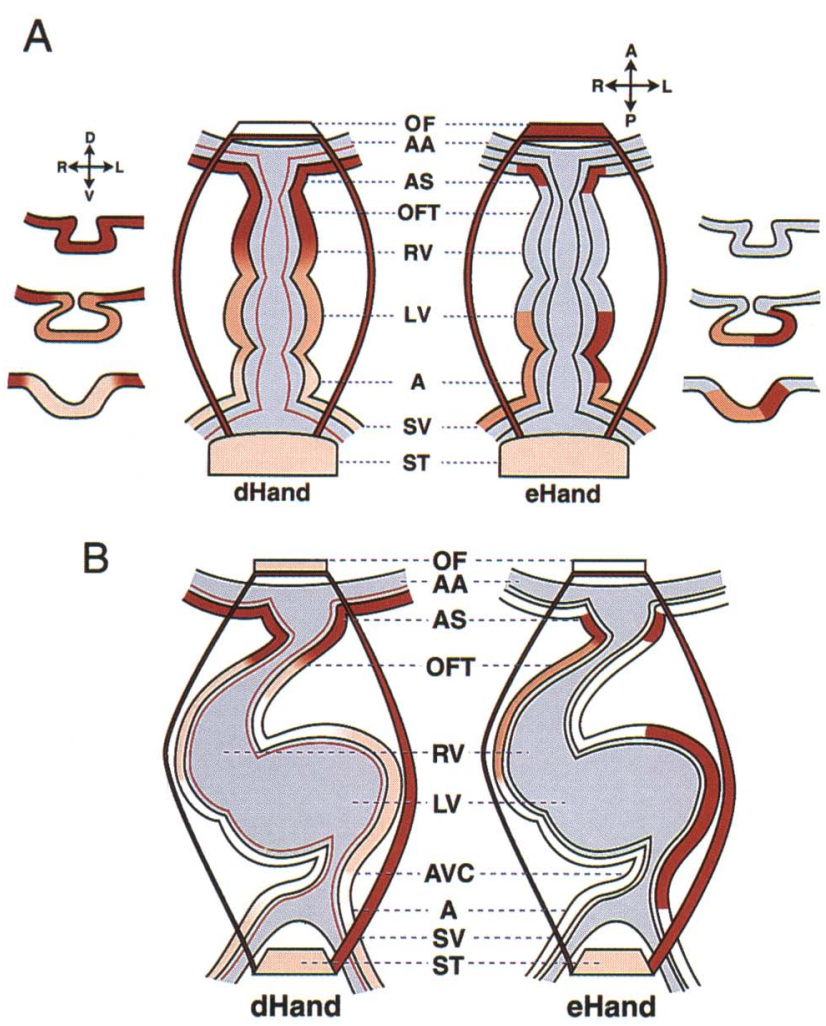

Figure 9. Summary of $e H a n d$ and $d$ Hand expression patterns in linear and looping heart tubes. The intensity of red shading indicates the approximate relative in situ hybridization signal strength detected in whole-mount embryos or sections. $|A|$ Ventral views of expression patterns in the linear heart tube and surrounding pericardium, oral cavity, and septum transversum, accompanied by the expression profile in schematic transverse sections at three anterior/posterior levels. Compasses orient

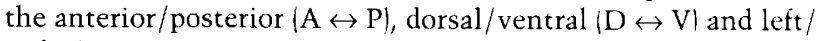
right $(\mathrm{L} \leftrightarrow \mathrm{R}$ )axes. $|B|$ Ventral view of expression patterns in the looping heart and surrounding tissues. (A) Atrium; $|\mathrm{AA}|$ aortic arch; (AS) aortic sac; (AVC) atrioventricular canal; (OF) oral cavity floor; (OFT) outflow tract; (LV) left ventricle RV) right ventricle; $(S T)$ septum transversum; $(S V)$ sinus venosa.

expression was symmetrical in the $L / R$ axis, but restricted to the ventral wall of myocardium, betraying for the first time a dorsal/ventral $(\mathrm{D} / \mathrm{V})$ patterning in the heart tube. Although $\mathrm{D} / \mathrm{V}$ differences have not been reported with other cardiac markers, patterning in this axis may be important for morphogenetic or myogenic development of the organ.

The most striking feature of the $e$ Hand caudal domain was the transition from bilateral to strictly left-sided expression. These patterns appeared distinct and several lines of evidence suggest that the transition between them is not a trivial consequence of torsion or other cellular movements associated with looping. First, evolution of a left-sided pattern through torsion would be at odds with the rightward movement of ventral myocytes observed in the chick (Stalsberg 1969). Second, in the linear heart tube, we saw coexistence of bilateral and enhanced left-sided expression (Fig. 4E), demonstrating that the left-sided pattern does not simply derive from rearrangement of the bilateral pattern. Third, the leftsided pattern extended more dorsally than the bilateral pattern (Fig. 4D). Fourth, even after the bilateral pattern had faded from the atrial chamber, a left-sided atrial expression persisted there for a time, before withdrawing to a boundary within the A/V canal (Figs. 2D and 4I). Atrial development differs substantially from that of the ventricles. Presumptive left and right ventricular chambers originate as neighbors along the $\mathrm{A} / \mathrm{P}$ axis, and assume a $\mathrm{L} / \mathrm{R}$ relationship later, a consequence of looping (Stalsberg and DeHaan 1969) (see Fig. 1). Left and right atria, on the other hand, have a $\mathrm{L} / \mathrm{R}$ relationship at the outset and are not rearranged by looping. Therefore, eHand expression exclusively in the left atrium (Figs. 2D and 4I), even transiently, would appear to betray a genuine $L / R$ asymmetry. Finally, bilateral expression could be distinguished from left-sided expression in Nkx2-5 mutant hearts - the bilateral pattern was established normally, albeit weakly, but left-sided expression was abolished totally. The data strongly suggest that there are two distinct but temporally overlapping modes of $e$ Hand expression in the caudal region of the heart - the first, bilateral, which fades; the second, left-sided. Although complex, the patterns reveal an important interface between the forming heart and the embryonic L/R asymmetry system, and provide the first clues to intrinsic genetic control of cardiac looping.

The sharp boundary at the outflow side of the eHand expression pattern in the left ventricle is noteworthy (Fig. 3H). This boundary is clearly within the left ventricle and does not correspond to the interventricular sulcus. The same boundary seems to be respected by the bilateral $e$ Hand pattern in the linear heart tube (Fig. 3E), suggesting that it is established early as a result of $\mathrm{A} / \mathrm{P}$ patterning within the heart. Therefore, left-sided $e H$ and expression in the ventricle is a product of both $\mathrm{A} / \mathrm{P}$ and $L / R$ patterning systems. Left and right ventricular chamber-specific patterns have been seen before, some exclusively from transgenes but not their endogenous counterparts (Zeller et al. 1987; Lyons 1994; Kelly et al. 1995; Moessler et al. 1996; Ross et al. 1996). Currently, it is not clear how these patterns relate to $A / P$ or to $L / R$ information, but the partial patterns seen in transgenic mice suggest a modular construction for the cis-regulatory elements of myofilament genes, with left and right chamberspecific enhancers. eHand may well participate in myogenic as well as morphogenic development of the heart. If this is true, then myogenic specialization of the ventricles would depend on $\mathrm{L} / \mathrm{R}$, as well as $\mathrm{A} / \mathrm{P}$, information.

\section{dHand expression}

$d H a n d$ was initially expressed throughout the cardiac crescent from the beginning of myogenic differentiation, before becoming substantially down-regulated in all but the aortic sac region of the looped heart (Fig. 9). In the linear heart tube, a gradient of $d$ Hand transcripts in myocardium was clearly evident (Fig. 7A-F). Although the gradient was transient, it may have a role in setting 
boundaries in the developing heart-targeted interruption of the $d H$ and gene leads to a specific loss of the right ventricular chamber (Srivastava et al. 1997).

For the most part, dHand expression in the cardiac region was symmetrical in the $\mathrm{L} / \mathrm{R}$ axis. However, the apparent right-sided expression of $d H$ and (and $e H a n d$ ) in the bulbotruncus of the looping heart could reflect an intrinsic $L / R$ asymmetry, although because these patterns appear only after the beginning of looping, this is difficult to assess and other interpretations are possible. For example, as noted above, rightward torsional movements, known to occur in the chick (Stalsberg 1969), could rearrange previously symmetrical patterns, or right-sided expression could be produced by an interaction between myocardium and pericardium on the outer (rightward) surface of the heart, where contact is most intimate.

\section{$L / R$ asymmetry and cardiac looping}

Cardiac looping is generally discussed as a rightward bending of the ventricular region of the heart. We have shown that the caudal region of the mouse heart develops asymmetrically before overt rightward looping, leading to a leftward displacement of the heart tube axis (Fig.1). Caudal asymmetry has been noted previously in human and chick hearts (de Vries and Saunders 1962; Orts Llorca 1970) and a leftward shift, similar to that described here for the mouse, occurs in the zebrafish (J.-N.Chen and M. Fishman, pers. comm.). The leftward shift may be driven, in part, by asymmetric proliferation, as more cells from the left progenitor pool contribute to the caudal heart in chickens (Stalsberg 1969) and the mitotic index is greatest in left caudal progenitors in mice (D. Bellomo and N. Brown, pers. comm.). This early asymmetry, occurring clearly before overt ventricular looping, may be a decisive event in determination of heart situs, as well as in the leftward positioning of the heart within the thoracic cavity.

Several genes have now been described in which expression patterns exhibit asymmetry in the $L / R$ axis /see Introduction!. Two of those, nodal and lefty, are expressed in left-sided lateral mesoderm extending cranially up to the developing heart progenitors and perhaps within precursors of sinus venosa, but not within those of the main body of the heart. This implies that the determination of situs involves, at least in part, inductive signaling to the heart from the left side. Not discounting the possibility of a right-sided (Stalsberg 1969; Hoyle et al. 1992; Isaac et al. 1997) or even earlier influence on heart looping (Smith et al. 1997), an appealing model at this time is that left-sided expression of nodal and/or lefty initiates the process resulting in caudal heart asymmetry, and drives some of the intrinsic cellular events of looping. We predict that eHand participates in the intrinsic response of the heart to left-sided signaling, controlling at least one aspect of the looping process. This model is consistent with our observation that inv/inv embryos, which show right-sided nodal and lefty expression and situs inversus (Lowe et al. 1996; Meno et al.
1996), also showed mirror-image reversal of caudal heart asymmetry and the side of $e H a n d$ expression.

\section{Hand expression in Nkx2-5 mutants}

To position eHand within genetic hierarchies controlling cardiac looping and laterality, we have examined its expression in different genetic models of cardiac dysmorphogenesis. The most significant finding is that leftsided $e H a n d$ expression was not observed in Nkx2-5 mutant hearts. We believe that loss of $e$ Hand expression in this model is not a secondary consequence of cardiac dysfunction. First, eHand was expressed normally in hearts of $\mathrm{Scl}^{-1-}$ embryos, which loop poorly because of secondary effects of haemopoietic deficiency. Furthermore, $d H a n d$ was expressed normally in both $N k \times 2-5^{-1-}$ and $\mathrm{Scl}^{-1-}$ mutants, arguing that $\mathrm{Hand}$ genes are not generally and nonspecifically sensitive to local conditions within dysfunctional hearts.

Although left-sided $e$ Hand expression was abolished

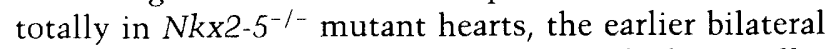
expression domain appeared to be established normally, albeit weakly. We can conclude that $e H a n d$ is transcriptionally competent in mutant myocardium, although it cannot be activated by $L / R$ information. This could be attributable to a defect in the L/R signaling system, or reflect a rather specific role for $\mathrm{Nkx} 2-5$ in the response of eHand to L/R signaling (see proposed models in Fig. 10). We suspect that mutant hearts are not blind to $L / R$ information, as they appear asymmetrical in their caudal region in a way resembling the caudal asymmetry described above for normal embryos (data not shown). Therefore, at the present time we favor a model in which Nkx2-5 and eHand lie in the same genetic pathway. In this model (Fig. 10A), Nkx2-5 could control eHand expression directly by interacting with the eHand enhancer module that responds to $L / R$ signaling, or indirectly through regulation of intermediate factors.

eHand was expressed ectopically in caudal pharyngeal floor endoderm in $N k \times 2-5$ mutants, a site where $N k \times 2-5$ is expressed in wild-type embryos at an earlier time (Lints et al. 1993). Therefore, Nkx2-5 regulates eHand expression both positively and negatively, depending on cellular context. A domain within the $\mathrm{Nkx} 2-5$ protein that has negative effects on transcription from synthetic reporter genes may be central to its dual role /Chen and Schwartz 1995).

\section{The role of Nkx2-5}

$N k \times 2-5$ is required for correct cardiac looping and leftsided $e$ Hand expression. Loss of eHand expression in Nkx2-5 mutant hearts is a likely cause of the looping defect. Our current findings on the mutant model demonstrate that $\mathrm{Nkx} 2-5$ serves both a myogenic and morphoregulatory role in murine heart development (Lyons et al. 1995; Harvey 1996; Zou et al. 1997), perhaps coordinating the two processes. In this respect, one role for $N k \times 2-5$ may be to capacitate the heart to respond to $L / R$ information. 
A

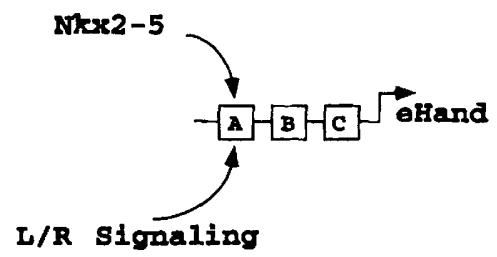

B

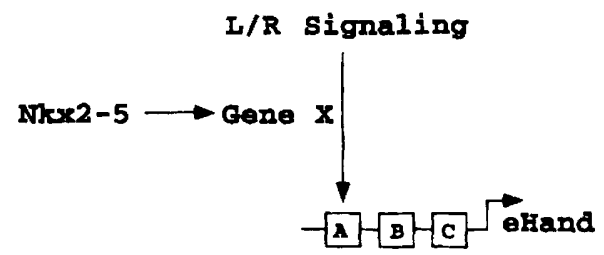

Figure 10. Alternative models that relate expression of $N k \times 2-5$ to that of eHand. $(A)$ The left/right (L/R) signaling pathway converges on one enhancer module $(\mathrm{A})$ of $e$ Hand cis-regulatory elements. Other modules (e.g., B and C) would drive expression in bilateral zones in heart and other tissues. $N k \times 2-5$ control is exerted at the level of module A. Transcription factors under $N k \times 2-5$ control, or the Nkx2-5 protein itself, collaborate with factors responding to $\mathrm{L} / \mathrm{R}$ signaling. $(B) N k \times 2-5$ controls expression in myocardium of a gene (Gene $\mathrm{X})$ necessary for a transducing signal from the $L / R$ asymmetry pathway. Gene $X$ may encode a ligand receptor or signal transduction intermediate. In this model, Nkx2-5 capacitates myocardium to respond to $\mathrm{L} / \mathrm{R}$ signaling.

In embryos in which $\mathrm{L} / \mathrm{R}$ axial information has been inhibited, the direction of heart looping is randomized (Levin et al. 1995; Hyatt et al. 1996). This information reveals an essential autonomy in cardiac looping, and suggests that the $\mathrm{L} / \mathrm{R}$ asymmetry pathway supplies only a bias for the choice of situs. In the absence of laterality signals, a stochastic event may otherwise determine its looping direction. In contrast, however, a whole spectrum of cardiac abnormalities results from defects in laterality (Burn 1991; Seo et al. 1992; Bowers et al. 1996), suggesting that establishment of asymmetry in the heart is often unsuccessful and that situs can be ambiguous. Because these cardiac defects are mostly serious and/or fatal, understanding the mechanism of looping is a central issue in cardiac biology. As initial steps toward this goal, we have identified eHand as the first intrinsic marker of lateral asymmetry in the mouse heart. Furthermore, we have shown that eHand expression falls under control of the homeobox gene Nkx2-5 in a genetic pathway responsive to laterality signals. Identification of the downstream targets of $e H$ and and $d H a n d$, and those of $N k \times 2-5$, will give us further insights into this important process in heart development.

\section{Materials and methods}

Whole mount in situ hybridization

Embryos between E7.0 and E11.5 were harvested by Caesarean section, dissected from decidua and membranes, and fixed overnight in $4 \%$ paraformaldehyde in PBS at $4^{\circ} \mathrm{C}$. Noon of the day of plugging was taken as embryonic stage E0.5. Gastrulating embryos (E7.0-E8.0) were staged as described previously (Downs and Davies 1993|. Whole-mount in situ hybridization using digoxygenin-labeled cRNA probes was performed essentially as described (Wilkinson 1992). The eHand probe corresponded to the full-length mouse cDNA (Cserjesi et al. 1995). The dHand probe corresponded to a 352 -bp fragment of the mouse cDNA covering amino acids $68-186$ of the dHand protein (E. Olson and D. Srivastava, unpubl.). $N k \times 2-5$ and $M L C 2 V$ probes were as previously described (Lints et al. 1993; Lyons et al. 1995). Embryos were photographed in PBS on a bed of agarose using a Wild dissecting microscope, in most cases with dark-field illumination.

\section{Sectioning of whole-mount embryos}

To facilitate handling, embryos were first embedded in $1 \%$ low melting temperature agarose, then subsequently dehydrated and embedded in paraffin wax. Microtome sections $(3-7 \mu \mathrm{m})$ were transferred to slides and counterstained with hematoxylin and eosin.

\section{Mouse strains and genotyping}

Normal embryos were from the CBA strain. Nkx2-5 mutant embryos on a mixed $129 / \mathrm{C} 57 \mathrm{Bl} / 6$ background were genotyped by PCR as described (Lyons et al. 1995) with modifications. The presence of a 220 -bp fragment from the wild-type allele was assessed using forward $\left(5^{\prime}\right.$-tgcagaaggcagtggagctggacaaagcc- $\left.3^{\prime}\right)$ and reverse $\left(5^{\prime}\right.$-ttgcacttgtagcgacggttctggaaccag- $\left.3^{\prime}\right)$ primers and a PCR program of $94^{\circ} \mathrm{C}, 30 \mathrm{sec} ; 62^{\circ} \mathrm{C}, 30 \mathrm{sec} ; 72^{\circ} \mathrm{C}, 30 \mathrm{sec}$ for 35 cycles. The presence of a 271-bp fragment from the targeted allele was assessed using forward $\left(5^{\prime}\right.$-gagctggacaaagccgagacg-3 $\left.3^{\prime}\right)$ and reverse $\left(5^{\prime}\right.$-ctaaagcgcatgctccagactg- $\left.3^{\prime}\right)$ primers and a PCR program of $94^{\circ} \mathrm{C}, 30 \mathrm{sec} ; 55^{\circ} \mathrm{C}, 30 \mathrm{sec} ; 72^{\circ} \mathrm{C}, 30 \mathrm{sec}$ for 35 cycles. Scl mutant embryos on a mixed $129 / \mathrm{C} 57 \mathrm{Bl} / 6$ background were genotyped using PCR as described (Robb et al. 1995) and by their pallid color and absence of blood cells in histological sections. The inv strain, OVE210, carrying a tyrosinase transgene insertion (Yokoyama et al. 1993), was maintained on a FVB/N (albinol background. Heterozygotes were recognized by their tan coat color. Homozygote embryos from heterozygote matings at $\sim$ E9.5 were identified by their situs inversus phenotype.

\section{Acknowledgments}

We thank Eric Olson and Deepak Srivastava for supplying Hand probes, and Daniela Bellomo and Nigel Brown for unpublished data. We are also indebted to Paul Overbeek for inv mice, Lorraine Robb for $S c l$ knockout mice, Steven Mihajlovic for histology, Jodi de Winter, Michelle Latimer, Sally-Anne Morris, and Linda Weiher for animal husbandry, and Daniela Bellomo, Nadia Rosenthal, and Patrick Tam for critique of the manuscript. This work was supported by the National Health and Medical Research Council (Australia), the National Heart Foundation of Australia (G94M4176), and the Human Frontiers Science Program (RG-308/95).

The publication costs of this article were defrayed in part by payment of page charges. This article must therefore be hereby marked "advertisement" in accordance with 18 USC section 1734 solely to indicate this fact. 


\section{References}

Bodmer, R. 1993. The gene tinman is required for specification of the heart and visceral muscles in Drosophila. Development 118: 719-729.

- 1995. Heart development in Drosophila and its relationship to vertebrates. Trends Cardiovasc. Med. 5: 21-28.

Boterenbrood, E.C. and P.D. Nieuwkoop. 1973. The formation of the mesoderm in urodelan amphibians. Part 5: Its regional induction by the endoderm. Wilhelm Roux's Arch. Dev. Biol. 173: 319-332.

Bowers, P.N., M. Brueckner, and H.J. Yost. 1996. Laterality disturbances. Prog. Pedintr. Cardiol. 6: 53-62.

Burn, J. 1991. Disturbance of morphological laterality in humans. In Biological asymmetry and handedness (ed. G.R. Bock and J. Marsh), pp. 282-299. John Wiley and Sons, New York, NY.

Challice, C.E. and S. Viragh. 1973. The architectural development of the early mammalian heart. Tissue Cell 6: 447-462.

Chambers, A.E., S. Kotecha, N. Towers, and T.J. Mohun. 1992. Muscle-specific expression of SRF-related genes in the early embryos of Xenopus laevis. Development 118: 1095-1106.

Chen, C.Y. and R.J. Schwartz. 1995. Identification of novel DNA binding targets and regulatory domains of a murine tinman homeodomain factor, Nkx-2.5. J. Biol. Chem. 270: 15628-15633.

Chen, J.-N. and M.C. Fishman. 1996. Zebrafish tinman homolog demarcates the heart field and initiates myocardial differentiation. Development 122: 3809-3816.

Cleaver, O.B., K.D. Patterson, and P.A. Krieg. 1996. Overexpres. sion of the tinman-related genes $X N k x-2.5$ and $X N k X-2.3$ in Xenopus embryos results in myocardial hyperplasia. Devel opment 122: 3549-3556.

Collignon, J., I. Varlet, and E.J. Robertson. 1996. Relationship between asymmetric nodal expression and the direction of embryonic turning. Nature 381: 155-158

Cross, J.C., M.L. Flannery, M.A. Blanar, E. Steingrimsson, N.A. Jenkins, N.G. Copeland, W.J. Rutter, and Z. Werb. 1995. Htx encodes a basic helix-loop-helix transcription factor that regulates trophoblast cell development. Development 121: 25132523.

Cserjesi, P., B. Brown, G.E. Lyons, and E.N. Olson. 1995. Expression of the novel basic helix-loop-helix gene $e H A N D$ in neural crest derivatives and extraembryonic membranes during mouse development. Dev. Biol. 170: 664-678.

de Vries, P.A. and C.M. Saunders. 1962. Development of the ventricles and spiral outflow tract in the human heart. Carnegie Inst. Wash. Contrib. Embryol. 37: 87-114.

DeHaan, R.L. 1965. Morphogenesis of the vertebrate heart. In Organogenesis (ed. R.L. DeHaan and H. Ursprung), pp. $377-$ 419. Holt, Rinehart \& Winston, New York, NY.

DeRuiter, M.C., R.E. Poelmann, I. VanderPlas-de Vries, M.M.T. Mentink, and A.C. Gittenberger-de Groot. 1992. The development of the myocardium and endocardium in mouse embryos. Anat. Embryol. 185: 461-473.

Downs, K.M. and T. Davies. 1993. Staging of gastrulating mouse embryos by morphological landmarks in the dissecting microscope. Development 118: 1255-1266.

Edmondson, D.G., G.E. Lyons, J.F. Martin, and E.N. Olson. 1994. Mef2 gene expression marks the cardiac and skeletal muscle lineages during mouse embryogenesis. Development 120: 1251-1263.

Evans, S., W. Yan, M.P. Murillo, J. Ponce, and N. Papalopulu. 1995. tinman, a Drosophila homeobox gene required for heart and visceral mesoderm specification, may be represented by a family of genes in vertebrates: $X N k X-2.3$, a second homologue of tinman. Development 121: 3889-3899.
Frasch, M. 1995. Induction of visceral and cardiac mesoderm by ectopic Dpp in the early Drosophila embryo. Nature 374: 464-467.

Fu, Y. and S. Izumo. 1995. Cardiac myogenesis: Overexpression of $X C s \times 2$ or XMEF2A in whole Xenopus embryos induces the precocious expression of XMHCa gene. Roux's Arch. Dev. Biol. 205: 198-202.

Gonzalez-Sanchez, A. and D. Bader. 1990. In vitro analysis of cardiac progenitor cell differentiation. Dev. Biol. 139: 197209.

Harvey, R.P. 1996. NK-2 homeobox genes and heart development. Dev. Biol. 178: 203-216.

Heikinheimo, M., J.M. Scandrett, and D.B. Wilson. 1994. Localization of transcription factor GATA-4 to regions of the mouse embryo involved in cardiac development. Dev. Biol. 164: 361-373.

Hollenberg, S.M., R. Sternglanz, P.F. Cheng, and H. Weintraub. 1995. Identification of a new family of tissue-specific basic helix-loop-helix proteins with a two hybrid system. Mol. Cell. Biol. 15: 3813-3822.

Hoyle, C., N.A. Brown, and L. Wolpert. 1992. Development of left/right handedness in the chick heart. Development 115: 1071-1078.

Hyatt, B.A., J.L. Lohr, and H.J. Yost. 1996. Initiation of vertebrate left-right axis formation by maternal Vgl. Nature 384: 62-65.

Icardo, J.M. 1984. The growing heart: An anatomical perspective. In Growth of the heart in health and disease (ed. R. Zakl, pp. 41-79. Raven Press, New York, NY.

Isaac, A., M.G. Sargent, and J. Cooke. 1997. Control of vertebrate left-right asymmetry by a Snail-related zinc finger gene. Science 275: 1301-1304.

Itasaki, N., H. Nakamura, H. Sumida, and M. Yasuda. 1991. Actin bundles on the right side in the caudal part of the heart tube play a role in dextro-looping in the embryonic chick heart. Anat. Embryol. 183: 29-39.

Jacobson, A.G. and A.K. Sater. 1988. Features of embryonic induction. Development 104: 341-359.

Kaufman, M.H. and V. Navaratnam. 1981. Early differentiation of the heart in mouse embryos. I. Anat. 133: 235-246.

Kelly, R., S. Alonso, S. Tajbakhsh, G. Cossu, and M.E. Buckingham. 1995. Myosin light chain 3F regulatory sequences confer regionalized cardiac and skeletal muscle expression in trasgenic mice I. Cell. Biol. 129: 383-396.

Komuro, I. and S. Izumo. 1993. Csx: A murine homeobox-containing gene specifically expressed in the developing heart. Proc. Natl. Acad. Sci. 90: 8145-8149.

Laverrière, A.C., C. MacNeill, C. Mueller, R.E. Peolmann, J.B.E. Burch, and T. Evans. 1994. GATA-4/5/6, a subfamily of three transcription factors transcribed in developing heart and gut. J. Biol. Chem. 269: 23177-23184.

Layton, W.M., M.D. Manasek, and D.M.D. Manasek. 1980. Cardiac looping in early iv/iv mouse embryos. In Etiology and morphogenesis of congenital heart disease (ed. R. Van Praghl, pp. 109-126. Futura, Mount Kisco, NY.

Levin, M., R.L. Johnson, C.D. Stern, M. Kuehn, and C. Tabin. 1995. A molecular pathway determining left-right asymmetry in chick embryogenesis. Cell 82: 803-814.

Lints, T.J., L.M. Parsons, L. Hartley, I. Lyons, and R.P. Harvey. 1993. $N k x-2.5$ : a novel murine homeobox gene expressed in early heart progenitor cells and their myogenic descendants. Development 119: 419-431.

Lowe, L.A., D.M. Supp, K. Sampath, T. Yokoyama, C.V.E. Wright, S.S. Potter, P. Overbeek, and M.R. Kuehn. 1996. Conserved left-right asymmetry of nodal expression and alterations in murine situs inversus. Nature 381: 158-161. 
Lyons, G.E. 1994. In situ analysis of the cardiac muscle gene program during embryogenesis. Trends Cardiovascular Med. 4: 70-77.

Lyons, I., L.M. Parsons, L. Hartley, R. Li, J.E. Andrews, L. Robb, and R.P. Harvey. 1995. Myogenic and morphogenetic defects in the heart tubes of murine embryos lacking the homeobox gene Nkx2-5. Genes \& Dev. 9: 1654-1666

Manasek, F.J. 1983. Control of early embryonic heart morphogenesis: A hypothesis. Ciba Found. Sym. 100: 4-19.

Manasek, F.J., B. Burnside, and R.E. Waterman. 1972. Myocardial cell shape change as a mechanism of embryonic heart looping. Dev. Biol. 29: 349-371.

Meno, C., Y. Saijoh, H. Fujii, M. Ikeda, T. Yokoyama, M. Yokoyama, Y. Toyada, and H. Hamada. 1996. Left-right asymmetric expression of the TGF $\beta$-family member lefty in mouse embryos. Nature 381: 151-155.

Moessler, H., M. Mericskay, Z. Li, S. Nagl, D. Paulin, and I.V. Small. 1996. The SM 22 promoter directs tissue-specific expression in arterial but not in venous or visceral smooth muscle cells in transgenic mice. Development 122: $2415-$ 2425.

Molkentin, J.D., A.B. Firulli, B.L. Black, J.F. Martin, C.M. Hustad, N. Copeland, N. Jenkins, G. Lyons, and E. Olson. 1996. MEF2B is a potent transactivator expressed in early myogenic lineages. Mol. Cell. Biol. 16: 3814-3824.

Montgomery, M.O., J. Litvin, A. Gonzalez-Sanchez, and D. Bader. 1994. Staging of commitment and differentiation of avian cardiac myocytes. Dev. Biol 164: 63-71.

Nascone, N. and M. Mercola. 1995. An inductive role for the endoderm in Xenopus cardiogenesis. Development 121: 515-523.

Orts-Llorca, F. 1970. Curvature of the heart: Its first appearance and determination. Acta Anat. 77: 454-468

Rawles, M.E. 1943. The heart forming area of the chick blastoderm. Physiol. Zool. 16: 22-41.

Robb, L., I. Lyons, R. Li, L. Hartley, F. Kontgen, R.P. Harvey, D. Metcalf, and C.G. Begley. 1995. Absence of yolk sac hematopoiesis from mice with a targeted disruption of the $\mathrm{Scl}$ gene. Proc. Nat1. Acad. Sci. 92: 7075-7079.

Rosenquist, G.C. 1966. A radioautographic study of labeled grafts in the chick blastoderm. Development from primitive streak stages to stage 12. Contrib. Embryol. Carnegie Inst. Washington 38: 71-110.

Ross, R.S., S. Navankasattusas, R.P. Harvey, and K.R. Chien. 1996. An HF-1a/HF-1b/MEF-2 combinatorial element confers cardiac ventricular specificity and establishes an anterior-posterior gradient of expression via an $N k \times 2-5$ independent pathway. Development 122: 1799-1809.

Schultheiss, T.M., S. Xydas, and A.B. Lassar. 1995. Induction of avian cardiac myogenesis by anterior endoderm. Development 121: 4203-4214.

Schultheiss, T.M., J.B.E. Burch, and A.B. Lassar. 1997. A role for bone morphogenetic proteins in the induction of cardiac myogenesis. Genes \& Dev. 11: 451-462.

Seo, J.W., N.A. Brown, S.Y. Ho, and R.H. Anderson. 1992. Abnormal laterality and congenital cardiac abnormalities. Relations of visceral and cardiac morphologies in the iv/iv mouse. Circulation 86: 642-650.

Smith, S.M., E.D. Dickman, R.P. Thompson, A.R. Sinning, A.M. Wunsch, and R.R. Markwald. 1997. Retinoic acid directs cardiac laterality and the expression of early markers of precardiac asymmetry. Dev. Biol. 182: 162-171.

Srivastava, D., P. Cserjesi, and E.N. Olson. 1995. A subclass of bHLH proteins required for cardiac morphogenesis. Science 270: 1995-1999.

Srivastava, D., T. Thomas, Q. Lin, D. Brown, and E.N. Olson.
1997. Regulation of cardiac msoderm and neural crest development by the bHLH transcription factor, dHAND. Nature Genet. (in press).

Stalsberg, H. 1969. The origin of heart asymmetry: Right and left contributions to the early chick embryo heart. Dev. Biol. 19: $109-129$.

-1970. Mechanism of dextral looping of the embryonic heart. Am. I. Cardiol. 25: 265-271.

Stalsberg, H. and R.L. DeHaan. 1969. The precardiac areas and formation of the tubular heart in the chick. Dev. Biol. 19: $128-159$.

Sugi, Y. and J. Lough. 1994. Anterior endoderm is a specific effector of terminal cardiac myocyte differentiation in cells from the embryonic heart forming region. Dev. Dyn. 200: $155-162$.

Tsuda, T., N. Philp, M.H. Zile, and K.K. Linask. 1996. Left-right asymmetric localization of flectin in the extracellular matrix during heart looping. Dev. Biol. 173: 39-50.

Wilkinson, D.G. 1992. Whole mount in situ hybridization of vertebrate embryos. In In situ hybridization: A practical approach (ed. D.G. Wilkinson), pp. 75-84. Oxford University Press, Oxford, UK.

Yokoyama, T., N.G. Copeland, N.A. Jenkins, C.A. Montgomery, F.F.B. Elder, and P.A. Overbeek. 1993. Reversal of left/ right asymmetry: A situs inversus mutant. Science 260: 679-682.

Yost, H.J. 1995. Vertebrate left-right development. Cell 82: 689 692.

Zeller, R., K.D. Bloch, R.S. Williams, R.J. Arceci, and C.E. Seidman. 1987. Localized expression of the atrial natriuretic factor gene during cardiac embryogenesis. Genes \& Dev. 1: 693-698.

Zou, Y., S. Evans, J. Chen, H.-C. Kou, R.P. Harvey, and K.R. Chien. 1997. CARP, a cardiac ankyrin repeat protein, is downstream in the $N k x 2-5$ homeobox gene pathway. Development 124: 793-804. 


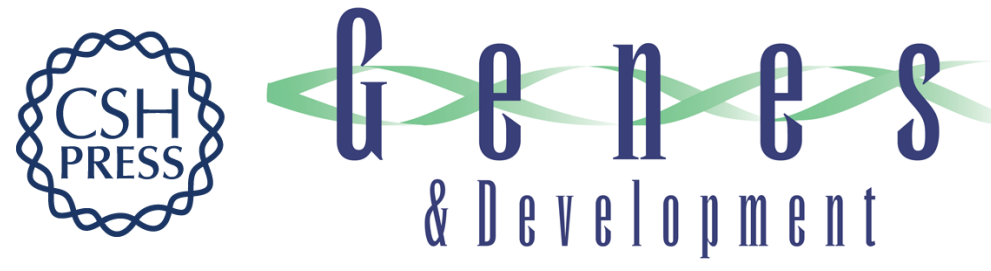

\section{Homeodomain factor Nkx2-5 controls left/right asymmetric expression of bHLH gene eHand during murine heart development.}

C Biben and R P Harvey

Genes Dev. 1997, 11:

Access the most recent version at doi:10.1101/gad.11.11.1357

References This article cites 63 articles, 29 of which can be accessed free at:

http://genesdev.cshlp.org/content/11/11/1357.full.html\#ref-list-1

License

Email Alerting

Service

Receive free email alerts when new articles cite this article - sign up in the box at the top right corner of the article or click here.

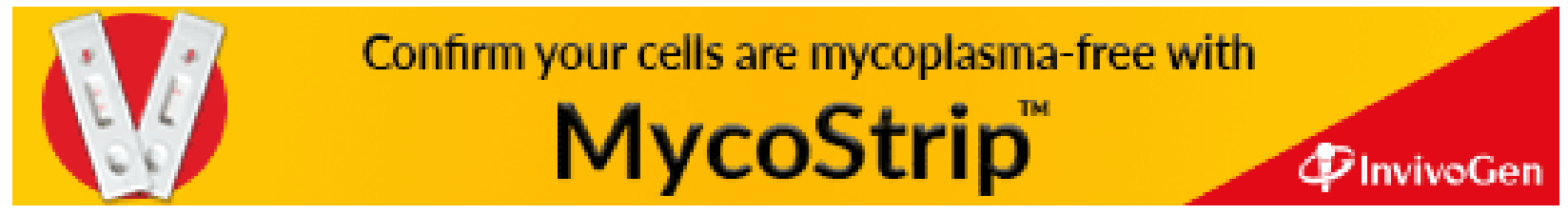

